\title{
Topological Force and Torque in Spin-Orbit Coupling System
}

\author{
F. J. Huang ${ }^{1,2}$, R. Qi ${ }^{1}$, Y. D. $\mathrm{Li}^{2}$, W. M. Liu ${ }^{1}$ \\ ${ }^{1}$ Beijing National Laboratory for Condensed Matter Physics, \\ Institute of Physics, Chinese Academy of Sciences, Beijing 100080, China and \\ ${ }^{2}$ School of Physics, Yunnan University, Kunming 650091, China
}

(Dated: July 6, 2018)

\begin{abstract}
The topological force and torque are investigated in the systems with spin-orbit coupling. It is demonstrated that the topological force and torque appears as a pure relativistic quantum effect in an electromagnetic field. The origin of both topological force and torque is the Zitterbewegung effect. Considering nonlinear behaviors of spin-orbit coupling, we address possible novel phenomena driven by the topological forces.
\end{abstract}

PACS numbers: 03.65.Pm, 72.10.Bg, 71.70.Ej

Recently, the spin-orbit coupling has become an interesting topic due to the spin Hall effect [1-5]. It provides an efficient route to generate and control quantum spin state electrically. Generally speaking, the spin-orbit coupling arises as relativistic quantum effect from the Dirac equation, and describes the interaction of the electron spin, momentum and electromagnetic field. In the system with spin-orbit coupling, the semiclassical equations of electron were studied recently, and some novel effects have been found [6-15]. However, in the systems with spin-orbit coupling, there remain some questions to be answered. As well known, the non-relativistic approximation of Dirac equation can be obtained from Foldy-Wouthuysen transformation. In the transformation, there exists a gauge potential in momentum space $[15,16]$, so one question is, what is the position operator in semiclassical equations? Since the position operator is the space-time parameter, its definition is significant. If we are uncapable of defining it correctly, we couldn't have the complete understanding for spin-orbit coupling.

In this paper, we investigate the gauge field of position operator in momentum space, and its related effects. Based on the dynamic continuity equation, we derive the quantum force and torque which contain two parts. The conventional part has the same form as in the classic electrodynamics, and the topological part originates from the spin-orbit coupling and other terms from relativistic quantum correction. We notice that the topological part has a close relation to the Zitterbewegung effect. For a two-dimensional system in a magnetic field, we propose that the topological force and torque can reveal more complicated phenomena.

Topological velocity The Dirac equation of electron with the wave function $\Psi=(\varphi, \chi)^{T}$ reads $i \hbar \frac{\partial}{\partial t} \Psi=H \Psi$, with the Hamiltonian $H=c \boldsymbol{\alpha} \cdot \boldsymbol{\pi}+\beta m c^{2}+V(\mathbf{x})$, where $\boldsymbol{\pi}=\mathbf{P}-\frac{e}{c} \mathbf{A}, \boldsymbol{\alpha}$ and $\beta$ are the $4 \times 4$ Dirac matrices, $V(\mathbf{x})=e \phi$ is a scalar potential, and $\mathbf{A}$ is a vector potential for a magnetic field, $\mathbf{B}=\nabla \times \mathbf{A}$. $m$ and $e$ are the electron mass and charge, and $c$ is the speed of light. To reveal the spin-orbit coupling in Dirac equation, we perform the Foldy-Wouthuysen transformation
$\Psi^{\prime}=U(\boldsymbol{\pi}) \Psi$, where $U(\boldsymbol{\pi})=e^{i S}$, it is a unitary transformation $[17,18]$. Choosing $S=-i(\beta \boldsymbol{\alpha} \cdot \boldsymbol{\pi} / 2 m c)$, and substituting it into the Dirac equation, we obtain the transformed Hamiltonian

$$
H^{\prime}=\beta m c^{2}+e^{i S} c \boldsymbol{\alpha} \cdot \boldsymbol{\pi} e^{-i S}+e^{i S} V\left(i \hbar \partial_{\mathbf{p}}\right) e^{-i S}-e^{i S} i \hbar \partial_{t} e^{-i S} .
$$

The scalar potential becomes $V(\mathbf{D})$ with covariant derivative defined by $\mathbf{D}=i \hbar \partial_{\mathbf{p}}+\mathcal{A}$, with the pure gauge potential $\mathcal{A}=i \hbar U(\boldsymbol{\pi})^{\dagger} \partial_{\mathbf{p}} U(\boldsymbol{\pi})$. By defining the covariant derivative, we have the position operator $\mathbf{X}=$ $i \hbar \partial_{\mathbf{p}}+\mathcal{A}$. The gauge potential $\mathcal{A}$ here is trivial. Neglecting the inter-band transition, and considering the adiabatic approximation, we find the non-trivial gauge potential

$$
\mathcal{A}=\frac{\hbar(\boldsymbol{\pi} \times \Sigma)}{4 m^{2} c^{2}}
$$

where $\Sigma=1 \otimes \boldsymbol{\sigma}$.

By expanding the Hamiltonian $H^{\prime}$, and taking $S^{\prime}=$ $\hbar e \boldsymbol{\alpha} \cdot \mathbf{E} / 4 m^{2} c^{3}$ to do another transformation $\Psi^{\prime \prime}=e^{i S^{\prime}} \Psi^{\prime}$, we get the non-relativist Hamiltonian

$$
\begin{aligned}
H_{s c h}= & \frac{\boldsymbol{\pi}^{2}}{2 m}+e \phi-\frac{e \hbar}{2 m c} \boldsymbol{\sigma} \cdot \mathbf{B}-\frac{\hbar^{2} e}{8 m^{2} c^{2}} \nabla \cdot \mathbf{E} \\
& -\frac{e \hbar}{8 m^{2} c^{2}} \boldsymbol{\sigma} \cdot(\mathbf{E} \times \boldsymbol{\pi}-\boldsymbol{\pi} \times \mathbf{E}),
\end{aligned}
$$

where the first two terms refer to kinetic energy and scalar potential, the third is the Zeeman energy, the fourth term is a Darwin term, refers to the correction of scalar potential, and the fifth term is so-called the spin-orbit coupling term. With this Hamiltonian, the Schrödinger equation with wave function $\psi$ then can be written as $i \hbar \frac{\partial}{\partial t} \psi=H_{s c h} \psi$, where $\psi=(1+(\boldsymbol{\sigma}$. $\left.\boldsymbol{\pi})^{2} / 8 m^{2} c^{2}\right) \varphi$ is the wave function $\varphi$ which have been performed unitary transformations. After the unitary transformations, the wave function $\chi \sim 1 / c^{3}$, so that we can neglect it. Based on this fact, we can regard the wave function $\psi$ have been done the normalization. In the Schrödinger equation of $\psi$, the position operator has 
been redefined by a covariant derivative in the momentum space $\mathbf{X}=\mathbf{D}=i \hbar \partial_{\mathbf{p}}+\mathcal{A}$, with the gauge potential $\mathcal{A}=\hbar(\boldsymbol{\pi} \times \boldsymbol{\sigma}) / 4 m^{2} c^{2}$.

We now turn our attention to the gauge potential induced by transformation $U(\boldsymbol{\pi})$. In our process, we consider the adiabatic approximation, so it is straightforward to interpret the gauge potential $\mathcal{A}$ as the non-Abelian Berry gauge potential, and it is Berry connection in momentum space, the Berry phase $\theta=\oint_{C} d \mathbf{p} \cdot \mathcal{A}$, where $C$ is loop in the momentum space. The Berry gauge potential describes the non-trivial geometry of the fiber bundle of the Hamiltonian eigenvectors over the phase space, it describes the influence of negative energy states in positive energy space.

Using $\mathcal{A}=\hbar(\boldsymbol{\pi} \times \boldsymbol{\sigma}) / 4 m^{2} c^{2}$, and the relation $F_{i j}=$ $\partial_{p_{i}} \mathcal{A}_{j}-\partial_{p_{j}} \mathcal{A}_{i}-(i / \hbar)\left[\mathcal{A}_{i}, \mathcal{A}_{j}\right]$, we get the non-Abelian Berry gauge curvature $F_{i j}=-\varepsilon_{i j k} \hbar\left(\sigma_{k} / 2 m^{2} c^{2}-(\boldsymbol{\sigma}\right.$. $\left.\boldsymbol{\pi}) \pi_{k} / 8 m^{4} c^{4}\right)$. Let $\lambda=-\frac{\hbar}{2} \sigma_{k}, \boldsymbol{\pi} \approx \mathbf{P}$, introducing a dual vector $F_{k}=\varepsilon_{i j k} F_{i j}$, then the gauge field can be rewritten as $F_{k}=\left(\lambda / m^{2} c^{2}\right)\left(1-\mathbf{P}^{2} / 4 m^{2} c^{2}\right)$, where the first term presents a monopole taking the radial length at the rest momentum $m c$ with its strength given by $\frac{\hbar}{2} \sigma_{k}$ in the momentum space, the second term is the higher order of correction. If we neglect the second term in $F_{k}$, then

$$
F_{k}=\frac{\lambda}{(m c)^{2}} .
$$

We note that this field only has two values $\pm \frac{\hbar}{2}$ in the momentum space, so the difference between this field and the ordinary field $F_{k}=\lambda / \mathbf{P}^{2}$ [19], is that this field is uniform. Obviously, this gauge field is a consequence of non-relativistic approximation $\mathbf{P} \ll m c$. In classical dynamics, the rest energy $m c^{2}$ does not affect the motion of particle in non-relativistic limit. However, in quantum mechanics, the rest momentum $m c$ induces a gauge field in the momentum space, then it will influence the motion of particle.

We notice that this gauge field can induce topological effects. Noting that after the unitary transformation, the covariant position operator of Dirac equation is defined by $\mathbf{X}$, and the commutator of covariant operator is not trivial: $\left[x_{i}, x_{j}\right]=i \hbar F_{i j}$. This relation is characteristic of the unitary transformation, it is this relation that induces the gauge field in non-relativistic approximation. Defining $\mathbf{x}=i \hbar \partial_{\mathbf{p}}$ as the canonical position operator, then $\mathbf{X}=\mathbf{x}+\mathcal{A}$, and the semiclassical equation of motion is $\dot{\mathbf{X}}=\dot{\mathbf{x}}+\dot{\pi} \times \mathcal{F}$, with $\mathcal{F}_{k}=F_{k} / 2$. Apparently, the last term of the equation above is a topological term, and results from the non-trivial commutator of covariant position operator. It acts as the Lorentz force in the momentum space.

By using the Hamiltonian (3), we define the velocity $\dot{\mathbf{X}}=\dot{\mathbf{x}}+\left(e \hbar / 4 m^{2} c^{2}\right)\left(\mathbf{E}+\frac{\boldsymbol{\pi}}{m c} \times \mathbf{B}\right) \times \boldsymbol{\sigma}$, where $\dot{\mathbf{x}}=\boldsymbol{\pi} / m+$ $\left(e \hbar / 4 m^{2} c^{2}\right) \mathbf{E} \times \boldsymbol{\sigma}$ is the conventional velocity. The second term in $\dot{\mathbf{X}}$ actually is a topological term, it is caused by the gauge field $F_{k}=\lambda /(m c)^{2}$, and we regard it as a topological velocity. Introducing the electron intrinsic magnetic moment $\hat{\mathbf{m}}=(e \hbar / 2 m c) \boldsymbol{\sigma}$, we can rewrite the velocity as

$$
\mathbf{v}=\tilde{\mathbf{v}}+\frac{\left(\mathbf{E}^{\prime} \times \hat{\mathbf{m}}\right) / 2 c}{m},
$$

where $\tilde{\mathbf{v}}=\dot{\mathbf{x}}$ is the conventional velocity operator which corresponds to position operator $\mathbf{x}, \mathbf{E}^{\prime}=\mathbf{E}+(\boldsymbol{\pi} / m c) \times$ $\mathbf{B}$ is the total electric field which affects an electron in its local coordinate frame. If we remind that the gauge field is induced by the interference between the negative and positive energy states, this topological term in Eq. (5) is contributed by the rest energy under the influence of negative states. Comparing with two velocities, we find the terms concern with $\mathbf{E}$ in Eq. (5) is twice that of conventional velocity. Besides, our definition adds a contribution of magnetic field. One can easily find that these differences between the two velocities is caused by the gauge field in momentum space.

Topological Force and Torque It is very interesting in the topological force which acts on the electron in an electromagnetic field. Let us start from the dynamic continuity equation. The dynamic continuity equation represents the change form of a physical quantify in spacetime, it always requires the existence of a source such as a force or a torque.

Denote by $\rho^{M}=\psi^{\dagger} \hat{\rho}^{M} \psi$ the momentum density, where $\hat{\rho}^{M}=m \mathbf{v}$. Using $\frac{\partial}{\partial t} \psi^{\dagger} \hat{\rho}^{M} \psi=\dot{\psi}^{\dagger} \hat{\rho}^{M} \psi+\psi^{\dagger} \hat{\rho}^{M} \dot{\psi}$ and $i \hbar \dot{\psi}=H_{s c h} \psi$, then we arrive at

$$
\dot{\rho}^{M}+\nabla \cdot \mathbf{J}^{M}=\mathbf{f},
$$

where $\mathbf{J}^{M}=\psi^{\dagger} \hat{\mathbf{J}}^{M} \psi=(1 / 2) \psi^{\dagger}\left\{\hat{\rho}^{M}, \tilde{\mathbf{v}}\right\} \psi$, and $\tilde{\mathbf{v}}$ is the conventional velocity. The force is given by $\mathbf{f}=$ $(1 / i \hbar)\left[\rho^{M}, H_{s c h}\right]=\mathbf{f}_{\text {con }}+\mathbf{f}_{\text {top }}$, where $\mathbf{f}_{\text {con }}$ is the conventional force which corresponds to the position operator $\mathbf{x}$, and $\mathbf{f}_{t o p}$ is the topological force. The conventional force can be read $\mathbf{f}_{c o n}=\rho^{C} \mathbf{E}+\left(\mathbf{J}^{C} \times \mathbf{B}\right) / c+$ $(\nabla \mathbf{B}) \cdot \mathbf{m}+(\nabla \mathbf{E}) \cdot \mathbf{P}+\left(e / 2 m c^{2}\right) \mathbf{E} \times\left(\hat{\mathbf{m}} \times \mathbf{B}^{\prime}\right)$, where $\rho^{C}=e \psi^{\dagger} \psi$ and $\mathbf{J}^{C}=e \psi^{\dagger} \tilde{\mathbf{v}} \psi$ are the charge and the current densities for $\mathbf{f}_{\text {con }} \cdot \mathbf{m}=\psi^{\dagger} \hat{\mathbf{m}} \psi$, and $\mathbf{P}$ is defined by $\mathbf{P}=\psi^{\dagger} \hat{\mathbf{P}} \psi=\psi^{\dagger}\left(\frac{\boldsymbol{\pi}}{2 m c} \times \hat{\mathbf{m}}\right) \psi$, it correlates with the electrical polarization induced by the spin-orbit coupling [10]. $\mathbf{B}^{\prime}=\mathbf{B}-(\boldsymbol{\pi} / 2 m c) \times \mathbf{E}$ presents the total magnetic field which an electron experiences in its local coordinate frame. Meanwhile, considering the topological velocity, the topological force can be found to be

$$
\mathbf{f}_{t o p}=\frac{e}{2 m c^{2}} \mathbf{E}^{\prime} \times\left(\hat{\mathbf{m}} \times \mathbf{B}^{\prime}\right) .
$$

It is clear that the topological force is an additional term to the conventional force, so that we can divide the total force into two parts: $\mathbf{f}=\mathbf{f}_{c l a}+\mathbf{f}_{s o}$ where $\mathbf{f}_{c l a}$ is the force the electron experiencing in classical motion, and $\mathbf{f}_{s o}$ is the force produced by the spin-orbit coupling. The 


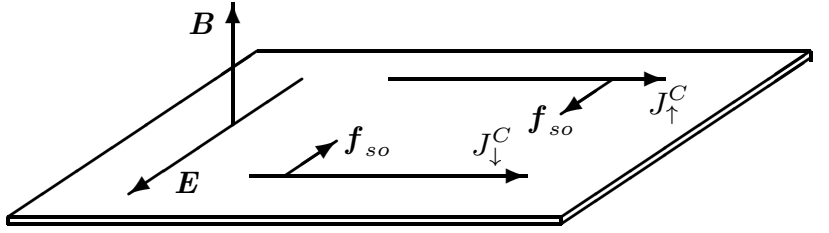

FIG. 1: The electrical field $\mathbf{E}$ and the magnetic field $\mathbf{B}$ exert the pure quantum forces $\boldsymbol{f}_{\text {so }}$ on the opposite spin polarized electrons, where $J_{\uparrow}^{C}$ and $J_{\downarrow}^{C}$ are charge currents with different spin polarization.

force $\mathbf{f}_{\text {cla }}$ can be written as $\mathbf{f}_{c l a}=\rho^{C} \mathbf{E}+\left(\mathbf{J}^{C} \times \mathbf{B}\right) / c+$ $(\nabla \mathbf{B}) \cdot \mathbf{m}+(\nabla \mathbf{E}) \cdot \mathbf{P}$, it is well known that the classical forces are Lorentz, "Stern-Gerlach", and electric dipole forces. All the forces above have correspondence forces in classical dynamics, so we combine them to a classical force $\mathbf{f}_{c l a}$.

The force $\mathbf{f}_{s o}$ can be read as $\mathbf{f}_{s o}=\left(e / m c^{2}\right) \mathbf{E}^{\prime \prime} \times(\hat{\mathbf{m}} \times$ $\left.\mathbf{B}^{\prime}\right)$, where $\mathbf{E}^{\prime \prime}=\mathbf{E}+(\boldsymbol{\pi} / 2 m c) \times \mathbf{B}$. To explore the physical meaning, we divide this force into two parts, $\mathbf{f}_{s o}=e \psi^{\dagger} \frac{\left(\mathbf{E}^{\prime \prime} \times \hat{\mathbf{m}}\right)}{m c^{2}} \times \mathbf{B}^{\prime} \psi+e \psi^{\dagger} \frac{\left(\mathbf{B}^{\prime} \times \mathbf{E}^{\prime \prime}\right)}{m c^{2}} \times \hat{\mathbf{m}} \psi$, where the first term indicates the electron experiencing an electrical field $\mathbf{E}^{\prime \prime \prime}=\left[\left(\mathbf{E}^{\prime \prime} \times \hat{\mathbf{m}}\right) / m c^{2}\right] \times \mathbf{B}^{\prime}$, and it is an electric force. One can find that the second term of $\mathbf{f}_{s o}$ is a pure quantum effect, since $\left(\mathbf{E} \times \mathbf{B}^{\prime}\right) / m c^{2}$ is dimensionless, this term does not concern with any classical quantify, it is a pure quantum force, as shown in Fig. 1.

When the magnetic field vanishes, the force $\mathbf{f}_{s o}$ reduces to $\mathbf{f}_{\text {ele }}=-\psi^{\dagger}\left(e / 2 m c^{2}\right)(\hat{\mathbf{m}} \cdot \mathbf{E})\left(\frac{\boldsymbol{\pi}}{m c} \times \mathbf{E}\right) \psi$. This force originates from the second term of $\mathbf{f}_{s o}$, so it is a pure quantum force, it can be used to explain the Zitterbewegung of the electron in spin-orbit coupling system $[7,8]$. The direction of this force is perpendicular to the electrical field, and to the velocity and the spin polarization direction, so it is a transverse force. When the electrical field vanishes, the force $\mathbf{f}_{s o}$ becomes $\mathbf{f}_{m a g}=-\psi^{\dagger}\left(e / 2 m c^{2}\right) \hat{\mathbf{m}} \cdot\left(\frac{\boldsymbol{\pi}}{m c} \times \mathbf{B}\right) \mathbf{B} \psi$, this force also originates from the second term of $\mathbf{f}_{s o}$, and it also is a pure quantum force. The direction of this force parallels with the magnetic field, and is perpendicular to the velocity and the spin polarization direction, so this force also is a transverse force. In fact, this force has duality with $\mathbf{f}_{\text {ele }}$, so it also can be known from the Zitterbewegung effect.

We now turn to the angular momentum continuity equation. Denoting $\rho^{A M}$ as the total angular momentum density, then $\rho^{A M}=\rho^{\mathcal{O}}+\rho^{S}$, where the orbit momentum density $\rho^{\mathcal{O}}=\psi^{\dagger} \mathbf{X} \times \hat{\rho}^{M} \psi$, the spin density $\rho^{S}=(\hbar / 2) \psi^{\dagger} \boldsymbol{\sigma} \psi$. Similar to the momentum continuity equation, we arrive at

$$
\dot{\rho}^{A M}+\nabla \cdot \mathbf{J}^{A M}=\mathbf{T} .
$$

The angular momentum current is defined by $\mathbf{J}^{A M}=$ $(1 / 2) \psi^{\dagger}\left\{\mathbf{X} \times \hat{\mathbf{J}}^{M}+(\hbar / 2) \boldsymbol{\sigma}, \tilde{\mathbf{v}}\right\} \psi$, where the first term is the orbit angular momentum current, and the second term is the spin current $\mathbf{J}^{\prime} S$. The total torque is given by
$\mathbf{T}=(1 / i \hbar)\left[\rho^{A M}, H_{s c h}\right]=\mathbf{T}_{c o n}+\mathbf{T}_{t o p}$, where $\mathbf{T}_{\text {con }}$ is the conventional torque which corresponds to the position operator $\mathbf{x}, \mathbf{T}_{\text {top }}$ is the topological torque. The conventional torque can be read $\mathbf{T}_{c o n}=\psi^{\dagger} \mathbf{x} \times \hat{\mathbf{f}} \psi+\mathbf{m} \times \mathbf{B}+$ $\mathbf{P} \times \mathbf{E}+\psi^{\dagger}(\boldsymbol{\pi} / 2 m c) \times(\mathbf{E} \times \hat{\mathbf{m}}) \psi$, where $\hat{\mathbf{f}}$ is an operator with $\mathbf{f}=\psi^{\dagger} \hat{\mathbf{f}} \psi$. Meanwhile, considering the topological velocity, the topological torque reads

$$
\mathbf{T}_{\text {top }}=\psi^{\dagger} \mathcal{A} \times \hat{\mathbf{f}} \psi+\psi^{\dagger} \mathbf{x} \times \hat{\mathbf{f}}_{t o p} \psi,
$$

where the first term concerns with the gauge potential, and the second term contributed by the topological force, so both two terms can be known from the Zitterbewegung effect.

Of cause, the total torque can be divided into the orbit torque $\mathbf{T}^{\mathcal{O}}$ and the spin torque $\mathbf{T}^{S}$. Combining the conventional and the topological torque, the orbit torque has the form $\mathbf{T}^{\mathcal{O}}=\psi^{\dagger} \mathbf{X} \times \hat{\mathbf{f}} \psi$. Obviously, $\mathbf{T}^{\mathcal{O}}$ has a corresponding torque in classical dynamics. The spin torque is given by $\mathbf{T}^{S}=\mathbf{m} \times \mathbf{B}-\psi^{\dagger} \hat{\mathbf{m}} \times\left(\frac{\boldsymbol{\pi}}{2 m c} \times \mathbf{E}\right) \psi$. The first term is contributed by the magnetic field. The second term is the influence of electric field $\mathbf{E}$, which can induce a magnetic field in electron's own coordinate frame, where $1 / 2$ is Thomas precession factor. These two terms can be found in Bargman-Michel-Telegdi Equation which describes the precession of the electron's spin [20].

Now we can write the spin continuity equation as $\dot{\rho}^{S}+$ $\nabla \cdot \mathbf{J}^{\prime} S=\mathbf{T}^{S}$, where the spin density is defined by $\rho^{S}=$ $(\hbar / 2) \psi^{\dagger} \boldsymbol{\sigma} \psi$, the spin torque is defined by $\mathbf{T}^{S}$. The spin current is $\mathbf{J}^{\prime} S=(\hbar / 4) \psi^{\dagger}\{\boldsymbol{\sigma}, \tilde{\mathbf{v}}\} \psi, \tilde{\mathbf{v}}$ is the conventional velocity operator.

Nonlinear Spin-Orbit Coupling We apply the previous results to a two-dimensional system under a weak magnetic field $\mathbf{B}=(0,0, B)$, with $\mathbf{A}=(0, x B, 0)$. The Hamiltonian reads $H_{2 D}=\pi^{2} / 2 m-\mu_{B} \boldsymbol{\sigma} \cdot \mathbf{B}+$ $(e F / 2)(\mathbf{E} \times \boldsymbol{\sigma}) \cdot \boldsymbol{\pi}+e E x$, where $\mu_{B}$ is Bohr magnetic momentum, $F=(\hbar / 2) /(m c)^{2}$ is the gauge field, the electrical field $\mathbf{E}=(E, 0,0)$. The topological velocity $v_{y}=\hbar k_{y} / m-\mu_{B} F B \sigma_{z} k_{y}-e F E \sigma_{z}$, where $k_{y}$ is wave vector. The spin current operators read $\hat{\mathbf{J}}_{y}^{z}=$ $\left(\hbar^{2} / 2 m\right) \sigma_{z} k_{y}-(\hbar / 2) \mu_{B} F B k_{y}-(\hbar / 2) e F E$, where the superscript presents the spin direction, the subscript denotes the direction of electron motion. It is found that the topological term here only concerns with momentum. In this case, the trajectories read $X=x+\hbar F k_{y} \sigma_{z} / 2$, $Y=y$. The spin current

$$
\mathbf{J}_{y}^{z}=\sigma_{s H} E,
$$

where the spin Hall coefficient $\sigma_{s H}=-(e / 4 \pi)(\delta+\delta \epsilon / 4-$ $\epsilon / 2)$, where $\delta=e B F / c, \epsilon=\hbar k_{F}^{2} F$ are dimensionless parameters, $k_{F}$ is the Fermi momentum. It is clear that the second term in the spin Hall coefficient $\sigma_{s H}$ is contributed by the gauge field.

For spin current $\mathbf{J}_{y}^{z}$, there is a pure quantum force perpendicular to the magnetic field, it is given by $f_{t o p}^{x}=$ 
$\mu_{B}\left(e E / m c^{2}\right) \psi^{\dagger} \sigma_{z} \psi B$. We can find that this force is proportional to the magnetic field. The directions of the spin polarization, the current and the force are perpendicular to each another. This force originates from the Zitterbewegung effect.

If we construct a spin-orbit coupling Hamiltonian as follows,

$$
H=\frac{\pi^{2}}{2 m}-\mu_{B} \boldsymbol{\sigma} \cdot \mathbf{B}+\eta(\boldsymbol{\pi} \times \boldsymbol{\theta}) \cdot \boldsymbol{\nu},
$$

where $\eta=\left(\hbar e / 4 m^{3} c^{3}\right) B$ is a spin-orbit coupling parameter, $\boldsymbol{\nu}$ is a unit direction which is along the $z$ direction, and $\boldsymbol{\theta}=\boldsymbol{\theta}(\boldsymbol{\pi}, \boldsymbol{\sigma})=\boldsymbol{\pi} \times \boldsymbol{\sigma}$ is a vector presenting spinorbit coupling types, we can also obtain the same velocity of the two-dimensional system above (the system has been eliminated the scalar potential). This indicates that the Hamiltonian $H_{2 D}$, which takes the gauge field into account, is equivalent to a system with more complex spin-orbit coupling. This equivalence illuminates that the gauge field can present more complicated spin-orbit coupling in spin-orbit coupling system, such as $\boldsymbol{\pi} \times \boldsymbol{\theta}$, with $\boldsymbol{\theta}=\boldsymbol{\pi} \times \boldsymbol{\sigma}$ or $(\boldsymbol{\pi} \cdot \boldsymbol{\sigma}) \mathbf{n}$, where $\mathbf{n}$ is a unit direction. In fact, Hamiltonian (11) is a generalization of Rashba model in which $\boldsymbol{\theta}(\boldsymbol{\pi}, \boldsymbol{\sigma})=\boldsymbol{\sigma}[21]$.

In the usual spin-orbit coupling system, the types of spin-orbit coupling are $\boldsymbol{\pi} \times \boldsymbol{\sigma}$ and $\boldsymbol{\pi} \cdot \boldsymbol{\sigma}$. They will exist as long as the spin-orbit coupling potential is not weak. However, these two types only present the first level of spin-orbit coupling, the types which we consider presenting the complicated level of spin-orbit coupling, such as $\boldsymbol{\pi} \times \boldsymbol{\theta}$, can also exist in a spin-orbit coupling system. Comparing with the first level, the complicated level of spin-orbit coupling is the coupling of the orbit and the first level. The first level is proportional to the orbit parameter $\boldsymbol{\pi}$, and the complicated level is proportional to the square of the orbit parameter, so one can find that the complicated level reflects the non-linear character of spin-orbit coupling system. In fact, there are not only the linear spin-orbit coupling, but also the non-linear spinorbit coupling in a spin-orbit coupling system. The first level of spin-orbit coupling such as $\boldsymbol{\pi} \times \boldsymbol{\sigma}$ or $\boldsymbol{\pi} \cdot \boldsymbol{\sigma}$ is the linear spin-orbit coupling, the complicated level such as $\boldsymbol{\pi} \times \boldsymbol{\theta}$ reflects the non-linear spin-orbit coupling. From this point, we say that the complicated level presents the non-linear character of a spin-orbit coupling system.

The significance of the topological force and torque lies in the fact that they provide a complete picture of describing the motion of a non-relativistic electron in a spinorbit coupling system. It is found that the topological force appears as a pure quantum effect when both electrical and magnetic fields exist in the system, while the topological torque originates from the topological force and a gauge potential. Through these force and torque, the Zitterbewegung effect can be understood deeply, the more complicated phenomena such as the nonlinear spinorbit coupling also can be revealed. This implies that the spin-orbit coupling can produce more novel effects.

We are grateful to S. Q. Shen, Y. G. Yao and J. R. Shi for helpful discussions. This work was supported by NSF of China under grant 10347001, 90403034, $90406017,60525417,10665003,10674163$, by the National Key Basic Research Special Foundation of China under 2005CB724508 and 2006CB921400.

[1] J. E. Hirsch, Phys. Rev. Lett. 83, 1834 (1999).

[2] S. Murakami, N. Nagaosa, and S. C. Zhang, Science 301, 1348 (2003).

[3] J. Sinova, D. Culcer, Q. Niu, N. A. Sinitsyn, T. Jungwirth, and A. H. MacDonald, Phys. Rev. Lett. 92, 126603 (2004).

[4] S. Q. Shen, M. Ma, X. C. Xie, and F. C. Zhang, Phys. Rev. Lett. 92, 256603 (2004).

[5] O. Entin-Wohlman, A. Aharony, Y. M. Galperin, V. I. Kozub, and V. Vinokur, Phys. Rev. Lett. 95, 086603 (2005).

[6] D. Culcer, J. Sinova, N. A. Sinitsyn, T. Jungwirth, A. H. MacDonald, and Q. Niu, Phys. Rev. Lett. 93, 046602 (2004).

[7] Z. F. Jiang, R. D. Li, Shou-Cheng Zhang, and W. M. Liu, Phys. Rev. B 72, 045201 (2005).

[8] S. Q. Shen, Phys. Rev. Lett. 95, 187203 (2005); B. K. Nikolic, L. P. Zarbo, and S. Welack, Phys. Rev. B 72, 075335 (2005).

[9] B. A. Bernevig and S. C. Zhang, Phys. Rev. Lett. 95, 016801 (2005).

[10] Y. Wang, K. Xia, Z. B. Su, and Z. S. Ma, Phys. Rev. Lett. 96, 066601 (2006).

[11] P. Q. Jin, Y. Q. Li, F. C. Zhang, J. Phys. A 39, 7115 (2006).

[12] E. I. Rashba, Phys. Rev. B 68, 241315 (2004); Phys. Rev. B 70, 161201 (2004).

[13] Q. F. Sun and X. C. Xie, Phys. Rev. B 72, 245305 (2005).

[14] J. Bolte and S. Keppeler, Ann. Phys. 274, 125 (1999); H. Spohn, Ann. Phys. 282, 420 (2000).

[15] K. Yu. Bliokh, Europhys. Lett. 72, 7 (2005).

[16] A. Bérard, H. Mohrbach, hep-th/0404165.

[17] L. L. Foldy and S. A. Wouthuysen, Phys. Rev. 78, 29 (1950).

[18] J. Bjorken, S. Drell, Relativistic Quantum Mechanics, (McGraw-Hill, 1964).

[19] M. V. Berry, Proc. R. Soc. London A 392, 45 (1984).

[20] V. Bargmann, L. Michel, and V. L. Telegdi, Phys. Rev. Lett. 2, 435 (1959).

[21] Y. A. Bychkov and E. I. Rashba, J. Phys. C 17, 6039 (1984). 